\title{
Terapia Breve Centrada en la Solución como modelo de terapia familiar de toxicómanos
}

ULIVI, G.

Responsable Médico SERT

\section{RESUMEN}

El modelo de Terapia Breve Centrada en la Solución presenta aspectos muy interesantes y útiles para el trabajo con los toxicómanos y sus familias. Las estrategias de enganche, haciendo propias las metas del paciente, estimulando la cooperación con los aspectos positivos y centrando la atención sobre modelos ligados a la solución más que al problema, hacen que éste se sienta inmediatamente protagonista de su cambio. Trabajar sobre los remedios, que se hacen aparecer a través de las preguntas adecuadas, permite al paciente activar un proceso que puede llevarle a la solución del problema. Todo esto crea una expectativa positiva para el futuro, muy útil para todo el sistema familiar y también para los especialistas, que se protegen del riesgo de resignarse rápidamente frente al paciente no motivado. El artículo expone las preguntas que permiten al drogodependiente reflexionar sobre los recursos que posee y motivarse para el cambio.

Palabras clave: terapia familiar, terapia breve, drogodependientes.

\section{EL MODELOTEÓRICO}

$\mathbf{L}$ a Terapia Breve Centrada sobre la Solución se está revelando como una aplicación clínica muy interesante para el tratamiento de los pacientes con problemas de consumo de drogas y de sus familias; los pronósticos sobre resultados son, además, muy buenos (de Shazer, 1982, 1986).

Esta terapia se basa en la idea de que nada ocurre por casualidad, y que es mucho más útil centrar la atención sobre las cosas positivas que ocurren que profundizar en los aspectos problemáticos.

El consumo de drogas no es una excepción; hay periodos en los que el sujeto decide no hacer uso de ellas. Desde el punto de vista de la Terapia Breve Centrada en la Solución el objetivo terapéutico se centra en ayudar al consumidor a descubrir y a utilizar los

\section{SUMMARY}

The model of brief therapy centred on the solution presents characteristics very interesting and useful in the work with the addicts and their families. The frameworks of coupling, making own the goals of the patient, stimulating the co-operation with the positive connotation and pointing on the solution's patterns rather than on those of the problem make the patient protagonist of the change immediately helping him to take out the resources that he has and he begins a process that allows to overcome the problem. This creates a positive prediction for the future very useful for all the family system and also for the operator that protects himself from the risk to resign early before patient not motivated. The article exposes the questions useful why addict reflect on the solution and give the reasons for change.

Key words: family therapy, brief psychotherapy, drug addict. modelos no problemáticos, con el fin de favorecer la repetición del modelo que contiene la solución.

Este modelo está claramente influido por Milton Erickson (Erickson 1978). En vez de detenerse sobre los modelos problemáticos ligados al pasado (que a la larga acaban centrándose en la búsqueda de errores y en el establecimiento de la culpa), el descubrimiento de las expectativas que existen respecto al consumo de las drogas y la proyección del consumidor hacia el futuro -cuando dicho consumo deje de representar un aspecto conflictivo- genera perspectivas favorables al cambio. Centrar la atención en los modelos de solución, como por ejemplo en lo que sucede los días en los que el joven decide no consumir drogas, se considera más importante que detenerse sobre cómo o cuántas veces hace uso de ellas. Cuando se descubre un modelo de solución, la tarea del terapeuta debe 
ser ayudar al paciente a encontrar los modos y las vías para repetirlo también en las situaciones en las que se corre el riesgo de recaída.

Ciertamente, no hay nada que funcione siempre, ni siquiera las metas construidas sobre los modelos del no consumo. Cuando los objetivos que se le proponen al paciente no coinciden con los suyos, es importante modular otros y trabajar con la idea de que es necesario hacer cosas distintas. Las tareas terapéuticas pueden ser vistas de otro modo, evitando repetir las que no funcionan. De hecho, la mayor parte de las veces, los problemas que tiene el paciente no son otra cosa que el resultado del hecho de que continúa haciendo, una y otra vez, cosas que no sirven, y si esto no se reconoce existe el riesgo de considerarlo como un sujeto resistente. El problema del consumo de drogas, en mayor medida que otros problemas, tiende a caer en la equivocación de que se propongan una y otra vez las mismas soluciones ineficaces que ya han sido intentadas y que no llevan a ningún sitio. Por eso es fácil cometer errores como el de dudar de la sinceridad de los comportamientos de los drogodependientes, reprendiéndoles continuamente, sermoneándoles y controlando sus historias para verificar su veracidad -esto no sólo aumenta la sensación de frustración, sino que también contribuye a aumentar los comportamientos de consumo y dificultar el pacto terapéutico.

Asegurarse la cooperación del paciente en el esfuerzo terapéutico debe ser, sobre todo para las personas que trabajan en la sanidad pública, un requisito fundamental y prioritario. La Terapia Breve Centrada en la Solución propone una visión sistemática e interactiva y proporciona a los terapeutas mucho espacio para instaurar relaciones positivas, incluso con pacientes que tienen fama de ser resistentes a los tratamientos. Se caracteriza por reconocer el valor del feedback positivo y valorar positivamente la importancia de los cambios pequeños y de sus efectos potenciales en el proceso terapéutico. De hecho, es más fácil, tanto para el paciente como para el terapeuta, centrar la atención sobre los cambios pequeños, y también es más fácil ver los resultados. Esto funciona especialmente bien con pacientes drogodependientes, que son sujetos que, por naturaleza, tienden a sentirse inseguros de sí mismos.

El descubrimiento de las propias capacidades y habilidades, de los propios intereses y éxitos, y así sucesivamente, representa no sólo una excelente técnica de enganche, sino que también evidencia los puntos fuertes tanto de los pacientes como de sus familiares.

Desde este punto de vista, el trabajo terapéutico es más un proceso de construcción de soluciones que de solución de los problemas. Este enfoque ofrece a los pacientes y a los terapeutas pistas para encontrar y utilizar las soluciones. Cuando se sienten estancados, hay veces en las que los pacientes no se dan cuenta de lo capaces que son, ni tampoco de que muchas veces han resuelto los problemas ellos solos. El rol del terapeuta se convierte, simplemente, en situarles en la dirección justa con el fin de que después puedan hacer algo autónomamente.

\section{LA MOTIVACIÓN COMO ELEMENTO CENTRAL DE LA VALORACIÓN}

Según el Modelo de la Terapia Breve Centrada en la Solución (TBCS) el aspecto más importante de la fase de valoración es el nivel de motivación del paciente. A los que abusan de las drogas se les suele ver como personas que ofrecen resistencia a los tratamientos y que son propensas al rechazo.

En concreto, muchas veces son los padres y las instituciones (las escuelas, los tribunales) los que obligan a los adolescentes a comenzar el tratamiento. Por ello es frecuente que los programas de tratamiento y las definiciones de los objetivos de quienes trabajan en la sanidad pública acaben convirtiendo a los terapeutas en agentes de control social. Y cuando surge una confusión entre la tarea terapéutica y el rol de control social también es frecuente que se culpe de ello al paciente, considerando, por ejemplo que no está motivado ni preparado para la terapia (Ulivi, 1996).

Como sucede en cualquier tipo de fenómeno interpersonal, la motivación del paciente para el cambio es el resultado de la interacción entre éste y el terapeuta. En vez de considerar la motivación del paciente como una cualidad innata y caracterológica que éste trae consigo a la terapia, es más útil considerarla como resultado de la interacción paciente-terapeuta (Miller, Rollnik, 1994). Esto es especialmente cierto cuando existe un elemento de coerción de por medio.

Normalmente los pacientes que abusan de las drogas están acostumbrados a ser amonestados repetidamente sobre los daños que éstas provocan: "perjudica a la salud", "destruye la vida", y cosas por el estilo: por eso es importante captar su atención por otros caminos. Hasta que un paciente que es, digamos que, reacio a las reprimendas reiteradas, no vea claramente los beneficios que puede reportarle el tratamiento, lo más seguro es que no coopere. Esto significa que si considera que la terapia puede ayudarle a obtener lo que más desea en ese momento, como por ejemplo, poder seguir estudiando, dejar de discutir con la familia, un poco más de libertad, la conquista de un afecto, y así sucesivamente, entonces cooperará. En las fases iniciales es importante trabajar sobre objetivos compartidos con el paciente, incluso en los casos en los que las metas que éste se propone puedan no ser los ideales o no reflejar directamente el problema real. 
La diferencia entre enviado y cliente representa un instrumento clínico muy útil y rápido para describir el modelo de la relación terapeuta-paciente. Las posiciones en las que se encuentra el paciente no son estáticas, sino más bien fluidas, y cambian de sesión en sesión. Que sea enviado o cliente depende del resultado de la relación terapeuta-paciente: el terapeuta tiene la posibilidad de transformar un enviado en cliente.

Los pacientes que consumen drogas y que son forzados a acudir a los tratamientos contra su propia voluntad se comportan como enviados. No sienten ningún interés por lo que se les ofrece. Lo único que parece interesarles es cumplir con la obligación de estar presente en las sesiones, pero no para tratar sobre el consumo de las drogas. Por ello es importante incrementar su cooperación y establecer relaciones terapéuticas, congratulándose con ellos por el hecho de que hayan acudido a una cita y siendo comprensivo en las discusiones de "estar tomándoles el pelo" y "que se les diga lo que tienen que hacer".

Cuando un drogodependiente rechaza participar en el tratamiento, o se está drogando en ese momento y no está en condiciones de afrontarlo, o los padres no son capaces de convencerle para acudir a la consulta, me resulta muy útil hacer venir a los otros miembros de la familia que no se drogan, generalmente a los padres. Cuando éstos son más clientes que sus hijos; es decir, cuando están más alterados o desesperados y más predispuestos y preparados para dar los pasos necesarios para producir el cambio, pueden modificar sus propios comportamientos no productivos respecto a sus hijos, elaborando, por lo tanto, modelos de interacción diferentes y positivos.

Transformar al enviado en cliente requiere investigar lo que es importante para el paciente, bien sea evitar la cárcel o intentar ayudarlo a la reinserción social, y es importante también utilizar su mismo lenguaje. Hay veces en que los padres o las figuras de referencia identifican el problema con la droga y, sin embargo, lo que más le interesa al paciente es, por ejemplo, poder usar el coche. En este caso habría que considerar que el paciente es un cliente que quiere comprar un coche y un enviado al que se quiere que se trate sobre la cuestión del abuso de drogas. Aceptar el objetivo de trabajar sobre la perspectiva de adquirir un vehículo puede llevar al paciente a darse cuenta de que el abuso de drogas interfiere con la meta propuesta.

Por ejemplo, profundizando sobre los intentos de solución que habían llevado a cabo los padres de una chica drogodependiente de 25 años, a la que llamaré Laura, durante la primera entrevista se puso en evidencia que se pasaban el día aconsejándola y sermoneándola, y que ya la habían enviado a varios profesionales privados con los que resistía un máximo de 3 ó 4 sesiones. Durante el primer encuentro Laura me había sorprendido por su disponibilidad para ir a diver- sos terapeutas, respondiendo así a la insistencia de su madre. Sintiéndome el último de la lista, con la única diferencia de que era el primero que trabajaba en un servicio público, me preguntaba qué podía hacer para no quemarme en pocas sesiones. Comprendí que lo que ella necesitaba era no tener más a los suyos pisándole los talones: la seguían para espiarla, la esperaban en el coche debajo de la casa de su novio y no le dejaban ni un minuto de autonomía. Cuando le comuniqué mi estupor por la disponibilidad que mostraba hacia sus padres y fui consciente de su objetivo conseguí que se enganchase: ya hace dos años que acude a las citas y lleva más de un año, después de un periodo de naltrexona, sin consumir heroína, como lo reflejan los reconocimientos a los que se somete cada dos semanas, que sigue haciendo delante de sus padres como pago de la libertad que ahora disfruta para poder moverse libremente por el mundo.

\section{DEFINICIÓN DE LOS OBJETIVOS DEL TRATA- MIENTO}

Cuando el objetivo del paciente se acepta como legítimo y válido, aunque pueda parecer que no esté relacionado con el consumo de las drogas, es muy frecuente que los pacientes resistentes empiecen a cooperar, convirtiéndose en clientes fuertemente implicados en la terapia. Los objetivos del tratamiento deben ser específicos y concretos. Por eso, el "quítame de encima a mis padres" debe desarrollarse a través de una descripción de lo que será la vida del paciente cuando éstos dejen de perseguirlo. Cuando los objetivos de los padres se diferencian de los del paciente (por ejemplo, los primeros quieren que abandone completamente el uso de drogas para que pueda ir mejor en la escuela mientras que el objetivo del segundo es dejarlas para poder tener más amigos), muchas veces es mejor y más productivo ver a los padres y a los hijos por separado. De hecho, viéndoles por separado el terapeuta puede contar con más espacio para desarrollar una solución que vaya bien a la familia.

En las situaciones en las que los miembros de la familia que se drogan son dos o más, resulta crucial combinar la valoración conjunta y separada para evaluar el objetivo de cada uno de ellos. Cuando todas las partes implicadas reconocen el consumo de las drogas y se convierten en clientes, pueden ser tratados simultáneamente, dado que entonces existe un objetivo común. De todos modos, cuando uno de los miembros de la familia es el cliente y otro es enviado, es más prudente empezar a trabajar sobre los procesos de cambio del primero, que a su vez pondrá en movimiento a toda la familia. 


\section{MODALIDADES OPERATIVAS EN NUESTRO CEN- TRO DE CONSULTA YTERAPIA FAMILIAR}

La principal tarea del terapeuta en la primera fase del tratamiento es enganchar al paciente aprendiendo su modo de ver el mundo y su lenguaje, descubriendo qué es lo más importante para él. Una vez que el objetivo del tratamiento ha quedado establecido, ayudar al paciente a realizarlo sitúa el problema de la droga como en un segundo plano. Hasta en los casos en los que el sujeto insiste en poner la atención sobre el problema, quizá porque está convencido de que cuanto más se hable de ello antes se resolverá, el apuntar sobre el estado futuro, cuando ya esté resuelto, le ayuda a ver que se puede conseguir una solución y que el cambio está al alcance de sus manos, sin necesidad de tomarse a broma su propia vida (Boscolo, Bertrando, 1993).

Una vez que ha comenzado el cambio, la tarea terapéutica es reforzar y vigilar otros cambios que estén empezando a cuajar.

Según la experiencia desarrollada en nuestro Centro de Consulta y Terapia Familiar, el trabajo en equipo, el espejo y la grabación en vídeo son instrumentos esenciales. Pensamos que si tras la tercera sesión de terapia individual no ha habido ningún cambio, el terapeuta puede consultar con el equipo, como uno de los recursos a los que acudir en la utilización práctica de este modelo. De nuestra experiencia en el uso del equipo hemos aprendido que las pausas que se destinan a la interpretación tienen un impacto dramático, porque aumentan las expectativas del paciente respecto a la terapia y éste siente un interés mucho mayor hacia cualquier cosa que diga el terapeuta. Nuestra experiencia es que si la aproximación del terapeuta al paciente es respetuosa, y si se toman en serio sus problemas, el rechazo de este último hacia el acercamiento del equipo y hacia la supervisión mediante el espejo bidireccional es mínimo.

En definitiva, el resultado más importante del esfuerzo terapéutico es potenciar al paciente, hacer que sea capaz de verse a sí mismo como protagonista de su propia vida y de ver que es capaz de vivir efectivamente.

\section{ESTRATEGIAS CLAVE DE LA INTERVENCIÓN}

Como hemos visto, nuestro tratamiento está orientado hacia el futuro, en la idea de que el modelo de solución es más importante que el modelo del problema. Por eso una de las tareas más importantes es la de formular preguntas para descubrir soluciones que funcionen y para construir el estado futuro cuando el problema ya esté resuelto.

\section{Las preguntas como intervención}

Las preguntas que siguen son ejemplos que utilizo bastante sistemáticamente y que pueden ser útiles como guía. Dado que la mayor parte de los pacientes y de los terapeutas no están acostumbrados a ellas, el uso efectivo de las mismas requerirá práctica y perseverancia.

\section{A. Preguntas para descubrir los momentos de no consumo}

"Veo con bastante claridad qué es lo que sucede cuando tienes el problema, pero ahora me gustaría considerar qué es lo que sucede cuando no estás consumiendo.

- ¿Hay días en los que no tienes problemas de consumo de drogas?

- ¿Cómo te explicas que ocurra esto?

- ¿Qué es lo que cambia de tu vida cuando no existe el problema?

- ¿Qué cosas distintas haces en esas ocasiones? ¿Y qué otras cosas?

-En tu opinión, ¿qué es lo que tendría que pasar para que esto sucediese más a menudo?

- ¿Qué es lo que podría convencer a tus padres (maestros, amigos, etc.) de que el problema se está resolviendo?

- ¿Cómo reaccionarían tus padres (maestros, amigos, etc.) al ver los cambios que empiezan a notar en ti?

- ¿Consigues no consumir bastante a menudo?

- ¿Durante cuánto tiempo consigues no consumir?

- ¿Qué es lo que tendrías que hacer para que sucediese esto?"

\section{B. Preguntas graduales}

Cuando la descripción de un problema y el objetivo del tratamiento son demasiado difusos o demasiado globales, las preguntas graduales ayudan a enfocarlos. También son instrumentos útiles para establecer qué tipo de inversión es necesaria para el cambio.

-"Sobre una escala de una a diez, con el diez de máximo y el uno de mínimo, ¿dónde situarías tu deseo de dejar de usar drogas?

- ¿Dónde te situarían tus familiares en esta escala?

- ¿Quién sería el más optimista? ¿Quién el más pesimista? ¿Y por qué piensas eso?

-Sobre la misma escala, ¿cuáles dirías que son tus posibilidades de conseguirlo? ¿Qué dirían tus padres? ¿Y tus profesores? ¿Y tus amigos? 
- ¿Qué tendrías que hacer para avanzar de 4 a 5 ? ¿Qué es lo que piensan tus familiares y amigos que tendrías que hacer?

\section{Preguntas hipotéticas o milagro}

Cuando un terapeuta no consigue a identificar momentos de no consumo y quiere obtener soluciones hipotéticas, o cuando los momentos de no consumo se han identificado y la tarea inmediata es establecer el objetivo, la pregunta milagro puede ser muy útil.

"Si esta noche sucediese un milagro mientras estás durmiendo y se resolviese tu problema:

- ¿Qué es lo que notarías a la mañana siguiente que te indicaría que ha sucedido un milagro? ¿Y qué otras cosas más?

- ¿Quién más se daría cuenta de que ha sucedido un milagro? ¿Cómo lo notaría?

- ¿Cuál es la diferencia que notarían tus padres en ti?

- ¿Cómo reaccionarían tus padres al notar los cambios que ha habido en ti?

- ¿Qué es lo que deberías hacer para conseguir que esto sucediese?

- ¿Cómo tendrías que empezar a comportarte, en tu opinión, para que pareciese que había ocurrido un milagro?".

\section{Preguntas en serie}

Cuando un paciente cuenta que nada le va bien, que da igual lo que intente, y persiste en la negación que de que esté sucediendo algo positivo en relación con sus propios padres o hijos, una buena técnica de enganche puede ser la de declararse de acuerdo temporalmente con la estima negativa haciendo "preguntas que afrontan la situación". Los ejemplos siguientes sirven para evidenciar al paciente que tiene los recursos y la fuerza necesarias para conseguir lo que desea y para mejorar su propia vida.

-Parece que el problema es verdaderamente muy serio. ¿Cómo es que las cosas no se agravan?

- ¿Qué es lo que haces (o tu familia) para que las cosas no vayan peor?

- ¿Qué tal te ha ido con las ayudas que has tenido? ¿Estarían de acuerdo tus padres (o hijos)?

- ¿Qué cosas fijas haces cuando las cosas son tan graves?

- ¿Qué serías capaz de hacer para que no sucediesen estas cosas?

- ¿Qué es lo que te indica que las cosas están empezando a ir un poco mejor?

-¿Qué es lo que te imaginas que harías después?

\section{E. Preguntas en serie pesimista}

Cuando un paciente insiste en que con su hijo (o con su padre) todo le va mal, y repite una y otra vez que hasta ese momento sólo ha obtenido resultados desastrosos, lo mejor engancharlo a este nivel planteando las siguientes preguntas:

"El problema que describes es verdaderamente serio y es necesario pensar sobre el porqué de que las cosas vayan tan mal.

-¿Cuál es la peor situación que puedes imaginar si las cosas no mejoran?

- ¿Cómo te imaginas que sería entonces su vida?

- ¿Qué es lo que piensas hacer ahora?

- ¿Quién lo pasaría peor si las cosas transcurren del peor modo posible? ¿Y después quién? ¿Algún otro?

Si el paciente se muestra un poco más optimista como respuesta a esta imagen tan pesimista, puede ser útil construir una solución planteando estas preguntas.

-Así que, ¿qué supondría para ti que las cosas vayan un poco mejor? ¿y qué otras cosas?

- ¿Quién es el primero que se da cuenta cuando empieza a producirse un cambio?

- ¿Cuál es la señal que indica que las cosas van un poco mejor?

-¿Qué supondría para ti hacer que eso sucediese?

\section{Felicitaciones}

Cuando el terapeuta destaca las cosas positivas que hace el paciente, como por ejemplo, acudir a la terapia a pesar de su escepticismo, mostrar buena voluntad para buscar un punto de encuentro con sus padres, querer evitar enredos con la justicia, y cosas de ese tipo, éste puede reaccionar con sorpresa y sentirse reconfortado. En muchos casos se trata de la primera vez que un profesional le dice cosas agradables, y probablemente también es la primera vez que alguien le ha prestado atención, especialmente si el paciente ha vivido una infancia escolar infeliz, ha tenido problemas con la policía, con la justicia, con sus padres, etc. El contenido de las felicitaciones no tiene por qué estar relacionado directamente con el problema en sí, sino con cualquier cosa que el paciente haga en su propia utilidad, o cualquier cosa que sea capaz de hacer, o a la que aspire. Lo más importante es evidenciarlo.

\section{El éxito futuro: fijar los objetivos}

Los objetivos claros, concretos y mensurables ayudan no sólo al terapeuta a comprender qué es lo que tiene que hacerse para resolver los problemas, 
sino que también aumentan las posibilidades de éxito del paciente. En el momento en que comienza la terapia la mayor parte de los pacientes se encuentran en un estado de opresión, confusión y frustración a causa de sus problemas que les impide especificar claramente cuáles son sus objetivos. Si se emplea el tiempo necesario para establecer unos objetivos estables y para fijar los elementos que permitan medir el éxito que se obtenga, los problemas parecen menores y menos opresivos. Massimiliano, un chico de 20 años que está en tratamiento por consumo de heroína y que lleva una vida desordenada a causa del consumo de drogas, ha expresado sus objetivos respondiendo a las preguntas milagro: no deberá consumir heroína como reacción a las disputas con su padres, empezará a trabajar, evitará a los muchachos de la plaza. Al terminar la sesión lo que ha sido capaz de construir a través de las respuestas de las preguntas puede ser sugerido como tarea para llegar a la solución.

\section{Valorar los periodos de no consumo transcurridos}

Hay pacientes que a menudo dejan pasar algunos días, una semana o dos, o quizás un mes sin consumir drogas. Y dado que hasta los viaje de miles de kilómetros comienzan paso a paso, el que haya habido uno o dos días de control o de abstinencia antes del inicio del tratamiento puede ser considerado como una señal de la capacidad del paciente para resolver el problema. La tarea del terapeuta consiste en examinar todas las cosas que el paciente haya hecho durante ese periodo de éxito. Y una vez que se integran en el repertorio de cosas sabidas, al paciente le resulta mucho más fácil empezarlas de nuevo.

\section{Enfocar la atención sobre el control}

Lo que nosotros proponemos es concentrarse en el control, no en la falta de control. Es decir, invitar al paciente a buscar qué es lo que hace en las ocasiones en las que pudiendo consumir las drogas consigue no hacerlo.

Este objetivo implica que el paciente vencerá la necesidad apremiante de hacer, en vez de esperar pasivamente a que sucedan las cosas. Cuando él o ella vuelven a ganar, esto implica que vencerán, y que sólo es cuestión de tiempo. La cuestión deja de centrarse en torno al hecho de si serán capaces de vencer o no.

\section{Reformular}

Dado que la percepción de las cosas es negociable, y dado que esta percepción de las cosas determina, por lo menos en parte, el comportamiento, muchas veces es mucho más útil ofrecer un punto de vista positivo de un acontecimiento que negativo.

Por ejemplo, Marcos, que tiene 34 años, llega a la sesión enfadado con sus padres porque siguen dudando de que haya dejado de consumir drogas. Después de escucharle un rato, he comentado por casualidad lo que deben quererle sus padres. Sorprendido al sentir una cosa de ese tipo, sintió curiosidad por sentirlas más veces. Le dije que por quererle tanto han sufrido mucho por su drogadicción, porque durante diez años, repitiendo una de sus frases, "nunca había bebido gaseosa" y que probablemente no consiguen hacerse a la idea de que esté bien por miedo a que vuelva a recaer, y que este problema de sus padres demostraba la fortaleza del vínculo que sentían hacia él. Después de quedarse reflexionando durante un rato muy largo, terminó confesando que sí, que estaba de acuerdo en que sus padres lo querían muchísimo.

\section{CONCLUSIONES}

El modelo de Terapia Breve Centrada en la Solución presenta aspectos muy interesantes y útiles para el trabajo con los toxicómanos y sus familias. Las estrategias de enganche, haciendo propias las metas del paciente, estimulando la cooperación con los aspectos positivos y centrando la atención sobre modelos ligados a la solución más que a los modelos de problema, hacen que éste se sienta inmediatamente protagonista de su cambio, ayudándole a utilizar sus propios recursos e insertando un proceso de posibilidades de superación del problema que crea una expectativa positiva para el futuro, muy útil para todo el sistema familiar y también para los especialistas, que se protegen del riesgo de resignarse rápidamente frente al paciente no motivado.

\section{BIBLIOGRAFÍA}

Boscolo L., Bertrando P., Presentificare il futuro. En Boscolo, L., Bertrando P., I tempi nel tempo. Bollati Boringhieri, Turín, pp. 170-202.

De Shazer S., (1982), Patterns of brief family therapy. Nueva York: Guilford Press.

De Shazer, S., (1978), Chiavi di soluzione in terapia breve, Astrolabio, Roma.

Guelfi, G.P., (1993), Autoaiuto e psicoterapia. Salute e Prevenzione 7-16, Roma.

Miller, W., Rollnick, S., (1994), I/ colloquio di motivazione. Ed. Erikson.

Ulivi, G., Aproccio motivazionale, modello sistemico-costruttivista: due riferimento per un lavoro integrato sul tossicodipendente e la sua famiglia. Personalità/dipendenze Vol. 2, Enero 1996, Mucchi ed., Milán. 